\title{
Tangence
}

\section{Écrire (dans) la ville : la Métropolis au féminin}

\section{Claudine Potvin}

Numéro 48, octobre 1995

Montréal et Vancouver : parcours urbains dans la littérature et le cinéma

URI : https://id.erudit.org/iderudit/025864ar

DOI : https://doi.org/10.7202/025864ar

Aller au sommaire du numéro

Éditeur(s)

Tangence

ISSN

0226-9554 (imprimé)

1710-0305 (numérique)

Découvrir la revue

Citer cet article

Potvin, C. (1995). Écrire (dans) la ville : la Métropolis au féminin. Tangence, (48),

84-96. https://doi.org/10.7202/025864ar d'utilisation que vous pouvez consulter en ligne.

https://apropos.erudit.org/fr/usagers/politique-dutilisation/ 


\section{Écrire (dans) la ville : la Métropolis au féminin Claudine Potvin}

La tendance des dernières années a montré que le féminisme, littéraire ou politique, privé ou public, risque toujours d'être évacué au nom d'un autre texte qui le comprend mal, revu et corrigé par une autre langue dans un autre lieu, ou tout simplement dépassé. Au cours de la dernière décennie, les pratiques culturelles semblent affirmer une forme de post-féminisme comme si l'autonomie du sujet féminin et son inscription dans le social et le symbolique étaient définitivement assurées. Or, le post-féminisme représente avant tout le regard d'un-e autre qui se situe déjà après les événements mais sans y avoir participé et qui ne retient que le grand dérangement d'une époque soi-disant révolue. De plus, au niveau de la praxis, le discours féministe s'est souvent vu accolé à celui du postmodernisme, les deux pensées ayant amorcé une tentative parallèle de déconstruction des discours uniformes, monologiques, unilatéraux et autoritaires d'une certaine Histoire. Dans un cas comme dans l'autre, il s'agissait de lever des interdits, d'éliminer des clôtures, de transformer des frontières, de pénétrer et de traverser des lignes interdites, de déplacer des ethnies, de redessiner et délimiter des territoires, de marquer autrement des propriétés, de renommer le sens, la direction, de faire signe ailleurs, de redéfinir le moi.

Si le postmodernisme surgit, selon Lyotard ${ }^{1}$, lorsque les grands récits de légitimation ne sont plus dignes de foi et que la vision du monde ne passe plus universellement par les concepts absolus de raison, de vérité, de progrès et de savoir, cette subversion postmoderne s'opère dorénavant au nom d'un principe de décentrement, de pluralité, de circulation, d'échange et de mouvance. Par ailleurs, la culture des femmes débouche sur une vaste entreprise d'exploration, de même que sur un questionnement et un renversement du discours social et politique officiel, dans l'espoir de créer un nouvel espace et une survie. C'est le processus que la théoricienne féministe américaine Alice Jardine a dénommé gynesis, soit la mise en mots de la femme comme

1 Jean-Françis Lyotard, La condition postmodeme, Paris, Minuit, 1979. 
procès intrinsèque de la condition postmoderne, c'est-à-dire la valorisation du féminin, de la femme et de son lien obligatoire, ou historique, aux nouveaux modes de pensée, d'écriture et de langage. L'objet produit par ce processus n'est ni une personne, ni une chose, mais un horizon, conclut Jardine, ce vers quoi le processus sémiotique tend, le gynema, ce dernier concept représentant un effet de lecture, a woman-in-effect, qui ne possède ni la stabilité ni l'identité ${ }^{2}$. Dans son article intitulé "Feminist Studies / Critical Studies: Issues, Terms, and Contexts", Teresa de Lauretis envisage à son tour une littérature féministe qui propose une politique de l'expérience, du vécu et de la vie quotidienne, qui pénètre la sphère publique de l'expression et de la pratique créatrice, déplaçant des hiérarchies esthétiques et des catégories génériques ${ }^{3}$. La critique féministe et l'écriture au féminin envisagent un sujet-femme et non plus seulement un effet-femme et elles réécrivent par conséquent la culture, le sexe et l'histoire.

La représentation urbaine dans l'écriture au féminin interroge précisément le concept même de représentation et met en place le rapport de l'écrivaine au discours de l'autre et du même. C'est dans la rue, le café, l'ascenseur, le bar, le métro, le musée, l'université, le cinéma, l'épicerie, de Montréal et d'ailleurs, autant de lieux d'où la femme parle et auxquels elle s'identifie, que se joue la rencontre femme-ville sous forme de rejet ou de complicité, ce que trois récits au féminin de la modernité québécoise exemplifient pleinement. Je renvoie ici à Picture Theory de Nicole Brossard, à la première nouvelle de L'bomme qui peignait Staline de France Théoret qui donne son titre au recueil et à La vie en prose de Yolande Villemaire, trois textes narratifs fort différents mais qui se rejoignent en partie dans la mise en forme d'un sujetfemme ${ }^{4}$. Cet essai cherche donc à cerner l'inscription textuelle de la ville dans trois fictions féministes et à en préciser la signification dans le cadre d'une réflexion sur l'écriture au féminin.

2 Alice A. Jardine, Gynesis. Configurations of Woman and Modernity, Ithaca \& Londres, Cornell University Press, 1985.

3 Teresa De Lauretis, "Feminist Studies / Critical Studies: Issues, Terms, and Contexts", T. de Lauretis (dir.), Feminist Studies/Critical Studies, Bloomington, Indiana University Press, 1986, p. 10.

4 Nicole Brossard, Picture Tbeory, Montréal, l'Hexagone, 1982; France Théoret, L homme qui peignait Staline, Montréal, Les herbes rouges, 1989; Yolande Villemaire, La vie en prose, Montréal, Les herbes rouges, 1980. Pour toute référence à ces ouvrages, j'indiquerai dorénavant la page entre parenthèses à la fin de la citation. 
86

Or, dans ces narrations de femmes écrites à partir du décor montréalais, si l'écriture s'appuie sur la ville, toile de fond et motif générateur du texte, c'est que la langue y circule tout en s'y cherchant un champ d'exploration. Bien que la ville tende parfois à exclure la femme, cette dernière n'en investit pas moins l'espace urbain dans et par la fiction. Lieu postmoderne par excellence, au sens où l'entend Scarpetta, c'est-à-dire en termes d'impureté, de mixage, de métissage, de courts-circuits, de rapts, de transferts, de recyclages, de détournements, de transpositions et de réappropriations ${ }^{5}$, le milieu urbain est ici à la fois producteur d'imaginaires, créateur de sens (orientation, signification) et inventeur de faux-fuyants; intertexte, la ville devient pour la femme écrivante un réseau de connivences et de complicités, lequel semble autoriser, voire provoquer l'écriture. La ville tend ainsi à déboucher (sur) le texte au moment même où celle qui y circule s'en détache, y inscrivant sa propre géographie citadine, inventant un langage différent pour la décrire, finalement traversant tous les sens uniques en direction opposée et transgressant les frontières d'un univers dorénavant trop restreint.

Dans sa nouvelle L'bomme qui peignait Staline, France Théoret construit son personnage féminin à partir d'une toile en apparence neutre. Le registre pictural contenu dans le titre se trouve doublement entouré d'une connotation masculine étouffante. Il est question de l'bomme sans identité, dans sa dimension générique d'une part, et d'un dictateur spécifique de l'autre. L'imparfait suggère le temps mort d'un certain discours idéologique fondé sur une vision du social et du politique qui a toujours exclu les femmes. Si le lecteur se reporte à l'ère stalinienne, il faut convenir que la peinture renvoie en ce sens à une forme de réalisme social essentiellement contraignante et au cadre esthétique patriarcal qui empêche la circulation du sujet féminin. Dans "L'avenir à l'infinitif", l'auteure se demande: "Comment assigner au féminin singulier un territoire de sujet quand on sait que toute place prise porte une charge mortifère dans cette société?" 6 Le paradigme du territoire reste à développer dans l'œuvre de France Théoret chez qui "Le récit s'organise dans un continuel excentrement: la vie, la mort, la séduction, la tyrannie, le quoti-

5 Guy Scarpetta, L'impureté, Paris, Grasset, 1985.

6 France Théoret, "L'avenir à l'infinitif", Vouloir la fiction, la modernité, La nouvelle barre du jour, 141, septembre 1984, p. 56. 
dien comblé, la solitude des rapports humains, plus encore le fait d'écrire tout en écrivant que "Les femmes sont incapables d'écrire "., ${ }^{7}$ La protagoniste narratrice de la nouvelle avoue ne pas avoir suivi l'évolution urbaine. Elle reçoit la ville comme un coup de vent. À la fois lieux de transformation, de liberté, corps de séduction et de répétition, banalité ou désolation, les villes de l'enfance et de l'âge adulte entrent dans la fiction de la fille comme un décor figé. "Ces lieux circonscrits, raconte Louise Aubert, fixés dans sa mémoire, devenaient métonymiques de l'architecture, de l'organisation sociale et du mode de vie de la majorité des gens, cette majorité à laquelle elle était consciente d'appartenir " (p. 24). Ici, le rapport de la fille à la rue en est un de nervosité, de crainte, voire de terreur comme lorsqu'elle se rendit au parc Lafontaine, un jour de fête, incapable de se mêler à la foule, de s'amuser, se tenant irrémédiablement à l'écart (p. 45).

Entre le travail et l'université, entre la chambre et le théâtre, seule la marche, lente et pénible, heureuse parfois, inscrit la narratrice dans la métropolis, dans la pensée, dans l'ouverture, dans le désir ${ }^{8}$. La petite chambre où se mettent en place la subjectivité et la rationalité féminines constitue un lieu serré, tendu, coincé, obscur, un lieu qui cache la ville, un point de fuite toutefois. Ainsi, de la cuisine et des chambres à coucher primitives de la jeune écolière à l'appartement de l'étudiante, Théoret dessine sur le mode répétitif une chambre exiguë et isolée qui ne fait pas quinze mètres carrés, aux meubles vétustes et aux murs d'anciennes couleurs pastel mal éclairés par une ampoule nue au milieu de la pièce. Une fenêtre, bouchée par un escalier de fer et habillée de rideaux opaques fermés en permanence, cache la vue de la réalité. C'est là que se créent l'expérience de la mémoire et la mémoire de l'expérience, c'est là que le poids du réel se fait le plus lourd. L'espace externe ne se vit qu'au milieu de la turbulence intérieure de la narratrice. C'est également dans ce lieu que surgit la parole signifiante d'un sujet féminin qui cherche avant tout à reconstruire l'espace de son être. À ce propos, Carole Massé commente en ces termes que "Désirer qu'une femme prenne sa

7 France Théoret, Entre raison et déraison, Montréal, Les herbes rouges, 1987, p. 79 .

8 L'importance du motif de la marche dans l'ouvre de France Théoret a été signalée maintes fois, entre autres par Pierre Nepveu dans aIntérieurs d'une pensée", Estuaire, 38, 1986, p. 23-29. 
88

place de sujet (et ne soit plus remise à "sa" place par quelque idéologie que ce soit), c'est accepter que cette femme puisse rejeter toute allégeance ou étiquette et ne désirer plus qu'être "?, de façon à ce que le masculin ne puisse plus biffer le féminin.

Absence, malaise, fuite, détour, le cheminement de l'héroïne /narratrice/ auteure inscrit le visible dans la citê, la marche du moi et de la subjectivité vers une forme de reconnaissance, la ligne d'un contour et la mémoire de l'écriture. La circulation limitée, réduite, bien souvent étouffée et censurée, n'en demeure pas moins une piste d'essai où la fille parle, bouge, écrit et observe, balise et délimite son territoire. L'espace y rejoint nécessairement le temps d'une cité idêale. "Soulever la rue" appartient à l'autre, à Mathieu, le compagnon qui contient en germe le fantasme du vainqueur, de l'envahisseur ou du conquérant. Maître des couleurs, celui-ci s'acharne à peindre Staline, alors que la fille, elle, trace la mémoire des mots déplaçant le réel au cœur de sa langue, travaillant le textuel pour éclater la réalité, permettre l'avènement de la subjectivité.

Sur un mode à la fois similaire et disjonctif, l'écriture de Brossard et de Villemaire appartient également à l'éclatement. Dans La vie en prose, Montréal se trouve sans arrêt récupéré par un autre récit qui passe par Hollywood, Los Angeles, San Francisco ou Venise. Les déplacements multiples suggérés par le roman empêchent l'écriture de se fixer sur un sujet. La ville, comme le texte, sera éclatée. D'une part, la réalité urbaine ne se perçoit qu'à travers un intertexte culturel qui colore et conditionne la narration: flash, photographies, titres de films, spectacles, allusions littéraires multiples, citations, invasion des univers dramatique et lyrique et de l'édition. De l'autre côté, l'éclatement spatial et temporel contenu dans la multiplicité des lieux (métros, miroirs, parcs, places publiques, Eaton's, Carré Saint-Louis, rue Saint-Denis, la Bibliothèque nationale, le Musée d'art contemporain, etc.) accuse la fragmentation verbale et structurale du récit. L'impression de cumul et d'excès ainsi créée produit un effet de mouvement incontrôlable. Le texte en dérive, automatiste, s'écrit précisément en dehors des lois de la circulation. Rapidement mis en circulation toutefois, bien que lent à s'écrire, le livre se fait dans la chaleur de la ville, la canicule, les

9 Carole Massé, "L'enjeu du sujet", Tessera, 9, automne 1990, p. 77. 
incendies même et dans le mouvement du spectacle (shows, performances, expositions). De plus, La vie en prose met en scène une écriture performative auto-réflexive. Le romanesque se construit au fur et à mesure qu'il se nomme. Ainsi les références au manuscrit, à l'édition, à la lecture ainsi que les échanges de lettres entre amies - les femmes se lisent entre elles, s'écrivent, se rencontrent, se racontent, discutent d'écriture mais surtout de tout et de rien - constituent autant de pré-textes à la fictionnalisation de l'espace urbain. En ce sens, sortir de la fiction supposerait sortir de la ville; le contraire s'avère tout aussi véridique. L'écriture de Villemaire, mélange de tous les styles et de tous les genres, préfigure le cosmopolitisme et le métissage d'une métropole repensée comme une utopie moderne. Dans son roman, l'auteure ouvre des frontières linguistiques et idéologiques, traverse des zones fermées au public, établit une folie du langage où les mots ne s'interrompent jamais, espèces de pianos mécaniques doublés d'un pianiste de concert, machines à écrire motorisées. De fait, la machine à écrire prend une place considérable au fur et à mesure que le récit progresse. L'écriture s'engendre elle-même, enchâ̂née, déchaînée, comme sous l'effet d'une drogue hallucinogène. La représentation de la ville dans le texte ne peut qu'échapper aux projections de la narratrice. Autonomie impossible car, d'une certaine façon, la ville n'a pas d'importance; seul compte le fait de s'y écrire, de l'écrire, de la décrire, de dire "je dans l'absence bienheureuse de toute conscience. (p. 243).

Dans Picture Theory, l'écriture traverse à nouveau l'espace de la ville et le corps s'y complaît. Là encore, profusion de lieux citadins: bars, hôtels, boulevards, cafés, cabarets, bibliothèques, musées, stationnements. Il s'agit d'occuper l'espace en utopie" (p. 185), écrit Brossard. Dans un numéro de La nouvelle barre du jour consacré à "La Femme et la ville", l'auteure écrivait que:

La ville fascine jusqu'à l'étourdissement. Réservoir inépuisable pour l'imagination, elle crée ses propres mythes de blasphèmes et de séduction. Elle soutient toutes les comparaisons s'offrant comme un éventail de sensations qui oscillent entre l'horreur et l'utopie, entre l'exaltation et la désintégration. La ville au crépuscule est un reflet de la cité, la polis des hommes. Ia ville, via l'image, via les corps comme un calcul mental ${ }^{10}$.

10 Nicole Brossard, "Pré(e)", La Femme et la Ville, La nouvelle barre du jour, 102 , avril 1981, p. 5. 
C'est en périphérie de la cité trajectoire, dans les terrains vagues et dans les territoires libres, que se construit l'utopie intégrale, la traversée des frontières; c'est là que s'élabore "ma" continent pour reprendre l'expression de Nicole Brossard.

Dans sa préface à ce roman poétique, Louise Forsyth souligne que :

On se tromperait si on voyait l'utopie de Nicole Brossard comme un lieu matériel et stable qu'elle rêve de réaliser. $\mathrm{Au}$ contraire, cette utopie est dynamique, mobile et en mutation. Comme l'hologramme elle n'est que l'énergie intense: forme vitale et image cohérente. Elle est l'abstraction dynamique, l'énergie psychique, l'émotion concentrée. En en empruntant l'élan toute femme prend sa place, à sa propre manière dans l'histoire de l'espèce, fait sens de sa quête ouverte et in-finie de la réalité. ${ }^{11}$

Selon Louis Marin, la fonction de l'utopie est transgressive: c'est une critique idéologique de l'idéologie ${ }^{12}$. Darko Suvin, pour sa part, complète la définition en utilisant le concept de jeu "mené par quelqu'un qui a le sens des possibles autres que ceux de la nature, surtout des possibles parallèles "13. Barbara Godard a commenté ces travaux, ajoutant que l'utopie sous-entend l'idée du double soit, "la projection métaphorique de la réalité "ailleurs" ", et "le déplacement métonymique des structures de la société contemporaine" ${ }^{14}$. Les écrits utopistes ne construisent pas toujours une fiction utopique mais réalisent quelquefois une fiction du réel basée sur la représentation des faits. Les nouvelles utopies, par ailleurs, ne sont pas des endroits fixes ou statiques. Selon Northrop Frye, elles ne seraient pas des cités rationnelles pensées par un philosophe mais localisées autant dans le corps

11 Louise H. Forsyth, "Préface", Picture Tbeory de Nicole Brossard, op. cit., p. $19-20$.

12 Louis Marin, Utopics: The Semiological Play of Textual Spaces, traduit par A. Volrath, Atlantic Highlands, N.J., Humanities Press International, 1984, p. 78. Titre original: Utopiques: Jeux d'espace. Les remarques qui suivent reprennent un commentaire sur l'utopie élaboré précédemment à propos d'un roman de Francine Noël; voir Claudine Potvin, "De l'Éden à Babel: écrire l'utopie", Voix et images, ${ }^{\circ}$ 53, hiver 1993, p. 299-300.

13 Darko Suvin, Metamorpboses of Science Fiction: The Poetics and History of a Genre, New Haven, Yale University Press, 1979, 53. Cité dans Barbara Godard, "En mémoire de l'avenir : les statégies de transformation dans la narration de Jovette Marchessault ", Voix et images, ${ }^{\circ} 49$, automne 1991, p. 105.

Barbara Godard, ibid., p. 105*106. 
que dans l'esprit, dans l'inconscient aussi bien que dans le conscient, dans la forêt et le désert comme sur l'autoroute et dans les édifices, dans le lit et le symposium ${ }^{15}$. En fait, comme l'ont signalé nombre de critiques, l'utopie, étymologiquement parlant, n'est pas un lieu. Non-lieu au centre de l'espace, l'utopie ne peut donc que se situer ailleurs.

Les deux romans de Brossard et de Villemaire se construisent précisément sur le mode utopique lié aux sphères urbaines qui constituent parallèlement des points d'embranchement ou de transfert et d'évasion. La rue, la place, le bar, le café, le restaurant, le métro, la bibliothèque, le musée seront perçus dans ce cadre avant tout comme des lieux limites où se concentre la charge utopique maximale, où tout est encore possible, inconnu: lieux de séductions, de désirs et d'angoisses. Foucault définit ces espaces autres comme "certains emplacements qui ont la curieuse propriété d'être en rapport avec tous les autres emplacements mais sur un mode tel qu'ils suspendent des rapports qui se trouvent, par eux, désignés, reflétés ou réfléchis " ${ }^{16}$. Il distingue deux types d'emplacements du genre, les utopies et les hétérotopies. Les premières seraient des emplacements sans lieu réel qui entretiennent avec les espaces réels de la société un rapport d'analogie directe ou inversée alors que les secondes constitueraient des lieux réels, des utopies réalisées. Le voyage, l'exil, la cité, le musée, le bateau, l'asile, l'institution, la clinique, la bibliothèque, la lecture, la frontière représenteraient alors des hétérotopies privilégiées de la société occidentale. La ville-frontière tend à intégrer tous les espaces mentionnés ici parce qu'elle crée un univers mixte: séparation et division de multiples mondes (ligne, limite, bord, cadre, zone, quartier, pôle, etc.) et, par extension, continuité et coupure.

Contrairement à la protagoniste de "L'homme qui peignait Staline" pour qui la frontière se limite au seuil de la porte, tout au moins en apparence, les héroïnes de La vie en prose et les femmes de Picture Theory traversent sans arrêt des lignes de nature géographique et culturelle: ville/mer, nord/sud, Québec/ États-Unis, Montréal/Paris-Rome, émotif/rationnel, pertes/quêtes,

15 Northrop Frye, "Varieties of Literary Utopias," F. E. Manuel (dir.), Utopias and Utopian Tbougbt, Boston/Cambridge, Houghton Mifflin Company/The Riverside Press, 1966, p. 49.

16 Michel Foucault, "Des espaces autres", conférence présentée à Paris le 14 mars 1967 et publiée dans A.M.C. Revue d'Architecture (1967). 
oppositions binaires où s'articule une sémiotique de l'espace centrée sur le mouvement et le passage. Le nom de Claire Dérive, objet de fantasme chez Brossard, suggère à lui seul le dérapage érotique, la fluidité, la dérive, ainsi qu'une forme de réflexion, de clarté, de luminosité et de lucidité. Effet albédo peut-être où se croisent l'imaginaire de la femme et l'énergie du rayonnement de la lumière sur une toile ou une feuille blanche.

Dans les textes de Brossard et de Villemaire, cet imaginaire féminin loge en partie à l'enseigne du musée inscrit dans la fiction de la ville. Lui-même frontière entre l'objet et sa représentation, reflet, projection en quelque sorte, le musée surgit dans la narration sous forme de "monument" historique, archéologique, folklorique, esthétique ou autre. "Original", il exige toutefois une fiction qui lui sert de commentaire et le justifie comme univers de représentation. Le corps-musée de la ville patriarcale renvoie par ailleurs à une scène de performance, de trans(e)formation, pur "propos de civilisation" ( $p, 41)$, dira Brossard. Musée et ville doivent éclater pour que la matière ainsi brisée s'assemble dans le livre car, ajoute cette dernière, "Mille fragments retombent sur mes épaules. De la matière partout, pièces d'identité : notes, lipstick, miroir, condom, clés, argent, mille fragments s'assemblent sous vos yeux dans le musée, dans le livre, il faut les voir venir" (p. 130). Le musée sera perçu dans ce contexte comme le véhicule d'une civilisation que le regard de la spectatrice transforme au moment où elle déambule dans les grandes salles vides qui ne parlent que de l'absence du féminin. Le nouveau discours muséographique inscrit dans la fiction propose alors une culture au féminin fondée non plus sur la valeur et l'échange économiques mais sur le dialogue. Eugenio Donato a signalé que si la fiction devait disparaître, il ne resterait rien dans le musée qu'un bric-àbrac, un ensemble de fragments d'objets sans valeur, incapables de se substituer aux objets eux-mêmes ou à leur représentation ${ }^{17}$. Adorno pour sa part souligne que le terme musée (mausolée, museal) décrit des objets auxquels l'observateur n'a plus de lien vital et qui sont en train de mourir ${ }^{18}$. "J'entre au Musée, remar-

17 Eugenio Donato, "The Museum's Furnace: Notes Toward a Contextual Reading of Bouvard and Pécuchet, "Josué V. Harari, (dir.), Textıal Strategies: Perspectives in Post-Structuralist Criticism, Ithaca, Cornell University Press, 1979 , p. 223.

18 Theodor W. Adorno, "Valéry Proust Museum", Prisms, Trad. Samuel \& Shierry Weber, Londres, Neville Spearman, 1967, p. 173. 
que encore Brossard. Une femme écarte les jambes pendant qu'un enfant flotte dans l'espace encore lié par le cordon et l'éclairage" (p. 56). Ainsi, dit-elle, entrer au musée comme on entre à l'hôtel, "pour comprendre le patriarcat" (p. 46), ce à quoi Louise Forsyth ajoute :

[...] c'est-à-dire à la frontière entre la réalité et le territoire imaginaire des femmes, lieu de l'ardeur. Elle est "celle qui écrit", consciente de la difficulté et de l'importance du travail de synthèse qu'elle fait dans la réalisation de l'utopie, la vision cohérente et intégrale de la femme. ${ }^{19}$

Sous le titre de Picture Theory, une théorie de l'image pénètre l'écran du langage (screen skin - screen - skintoo - screen skin utopia). D'une forme de l'ordinaire naît un vécu de femme, un penser au féminin.

Dans La vie en prose par contre, on ne va pas vraiment au musée pour l'exposition sinon pour s'y mettre en scène, se donner en spectacle, performer, "pour en évacuer toute réalité, bien sûr" (p. 287), y lit-on. Le roman tend à confondre ses lecteurs et ses lectrices en maintenant une perpétuelle ambiguité entre le réel et le fictif. Sur le mode de l'équivoque généralisée, c'est bel et bien le signifiant qui circule ici comme la femme dans la ville, apparemment dénué(e) de tout signifié. Ce livre séduit en ce qu'il évacue la mort. Il s'agit toujours de s'y représenter soi-même au seuil du plaisir, dans le monde éparpillé qui entoure les narratrices, un univers infiniment narrable. De la sorte, tout lieu urbain consacré, monumentalisé, sera investi et transformé; cette transgression s'opère bien entendu sous le signe de la démesure et du surplus. Au fil des pages, l'abondance ne raconte que le processus même d'une écriture gonflée, d'un embouteillage: traffic jam, refus du sens unique, ouverture de la cité de l'autre. Les femmes se promènent dans la fiction comme d'autres déambulent dans la ville, leurs manuscrits constituant les artères principales et secondaires d'une nouvelle histoire à raconter. Mais par où commencer, où finir cette lecture/écriture d'un quotidien essentiellement ex/centrique? Le mot, la ligne, la page et le livre forment les quadrilatères où la narratrice et l'écrivaine se sentent parfois encore coincées. Se pourrait-il que les femmes n'aient pas le sens de l'orientation? Désarmorcer les espaces urbains revient à sortir de 
94

la maison, descendre du trottoir, arpenter la nuit, s'approprier la rue, signer la fiction. "La ville est la mémoire de la machine, écrivait Yolande Villemaire dans "Les anges incognito", et il importe que nous l'investissions pour qu'on ne puisse jamais plus nous effacer de l'histoire. Et si on pleure de joie derrière nos lunettessoleil de star en forme de cueurs c'est qu'on sait maintenant qu'il sera désormais impossible de nous aliéner notre pouvoir. " 20

Le cri, l'excès et la complicité unissent ces femmes de fiction et les rapprochent d'une nouvelle Amérique, car l'utopie, "en commençant par le mot femme", selon Brossard, "n'allait pas assurer notre insertion dans la réalité mais [qu']un témoignage utopique de notre part pouvait stimuler en nous une qualité d'émotion propice à notre insertion dans l'histoire" (p. 102). Tout le rapport à la polis des hommes, à la cité des dieux, se fait par le biais de la loi et de l'ordre. Comme les cartes routières définissent ou indiquent la séparation et les frontières, le plan urbain reproduit les grandes voies de circulation qui entourent des territoires précis, des propriétés privées, des terrains publics, des zones réservées et des quartiers résidentiels, autant de lignes à traverser, autant d'interdits à transgresser à ses risques et périls. Dans ce contexte et dans un sens plus large, on pourrait évoquer le concept deborder writing, tel que développé par D. Emily Hicks à partir d'un corpus latino-américain. Selon la critique, cette écriture surgit d'une culture frontalière ou marginale dont une des principales caractéristiques réside dans le fait que la configuration des pratiques culturelles affecte la relation identitaire au territoire $^{21}$. La désintégration de l'ordre culturel, linguistique et politique (déterritorialisation également, dans certains cas, au sens où l'entendent Deleuze et Guattari d'appauvrissement de la langue des littératures mineures) ${ }^{22}$ caractérise l'écriture frontalière et accentue les différences entre les codes référentiels de deux ou plusieurs cultures au lieu de les effacer comme le souligne Régine Robin dans nombre de ses essais critiques. Finalement, les récits de frontière sont essentiellement décentrés. Semblable dans leurs pratiques à une littérature de frontière, la culture et l'écriture des

20 Yolande Villemaire, "Les anges incognito," La Femme et la Ville, La nouvelle barre du jonır, op. cit., p. 40.

21 Voir D. Emily Hicks, Border Writing. The Multidimensional Text, Minneapolis/ Oxford, University of Minnesota Press, 1991.

22 Voir à ce sujet Gilles Deleuze et Félix Guattari, Kafka, Paris, Éditions Minuit, 1975 , p. $29-50$. 
femmes se placent également dans une sorte d'hétérogène langagier $^{23}$. L'écriture s'avère un voyage d'exploration au cours duquel la femme tente de retrouver des pistes perdues et s'acharne à faire les cent pas au coeur de la langue en vue de créer de nouvelles traces et afin de se réinventer une mémoire collective. Néanmoins, la place des femmes reste toujours problématique, puisque marginalisée. Toutefois, la ville-frontière nie l'unicité et tend à déplacer les espaces ethnoculturels et à permettre des assemblages et des agencements différents, des aménagements complexes, l'altérité. Le texte/hologramme, brossardien ou autre, c'est le texte de la ville ouverte, déconstruite, renversée, magique, transparente, ludique. Selon D. Emily Hicks, de la même manière qu'une partie de l'hologramme peut produire une image complète, la métaphore de la frontière est capable de reproduire toute la culture à laquelle elle se réfère. Les métaphores de la frontière sont holographiques parce qu'elles re-créent l'ordre social dans son entier, bien qu'il ne s'agisse que d'un tout dans sa fragmentation ${ }^{24}$.

Bref, la représentation de la ville dans ces trois récits de France Théoret, Nicole Brossard et Yolande Villemaire rejoint cette dimension de l'écriture de frontière. Ces écrivaines pionnières ont précisément fait reculer à plus d'un titre le concept traditionnel de frontière gardée ou policière qui refoule l'étranger au lieu de l'accueillir et ce sur tous les plans (narratif, linguistique, sémique). Leurs écrits débouchent sur la promenade, la danse, la course, l'image, l'inscription du sujet dans le décor ou sur le panneau-réclame, car, déclare la narratrice de Picture Theory, "La ville est cet excès qui me prend comme une vitale exubérance...: j'avance, j'avance, se dit-elle, vers la répétition. J'avance, penset-elle très fébrilement pour ne pas s'arrêter devant une vitrine et y voir des mannequins enchaînées" (p. 49); l'avancée de la femme deviendra plus loin l'affirmation d'une victoire au moment où elle confirme que "Plongée au centre de la cité, je songerais à lever les yeux. Femme SKIN TRAJECTOIRE"(p. 184).

En conclusion, si la femme/narratrice/héroïne s'éparpille parfois dans le paysage urbain, observant les limites et les frontières de l'autre vision des choses, elle récupère ces effets de dissémina-

23 Voir Régine Robin, Le roman mémoriel. De l'bistoire à l'écriture du bors-lieu, Longueil, Le Préambule, 1989, p. 171.

24 D. Emily Hicks, op. cit., p. XXIX. 
96

tion au moyen de l'occupation systématique des lieux de parole. Écrire (dans) la ville pour les écrivaines suppose l'éclatement de la fiction et le fait, le désir, de se pencher sur le processus autoréflexif de la création, comme on fait couler de l'encre entre les fentes des édifices qui se cachent dans les mots. Entre le boulevard Saint-Joseph et la mer, entre les rues Cherrier et Mentana et entre New York et le Mexique, Montréal fait "signe" et passe dans le regard de la spectatrice comme un film à l'envers, d'autant plus séduisant que l'ordre n'y respecte plus le réel. Or, s'asseoir dans la ville, s'y installer d'une part, s'y soustraire de l'autre, y écrire surtour implique un double mouvement nécessaire: celui de s'inventer des pistes vierges pour pouvoir se fabriquer une ville à soi, un monde personnel qui contienne lui aussi une part du collectif. Il s'agit alors de faire siennes les longues marches dans la cité étrangère. Pour Théoret, Brossard et Villemaire, se balader dans la ville ne permet jamais que de retrouver la fiction et de vivre la "vie en (p)rose". Par extension, vouloir la fiction de la métropolis ou autre, c'est aussi vouloir la théorie à partir de laquelle elle s'amorce. Enfin, si la ville reste parfois le corps et le texte des autres, pour ces auteures, le charme s'opère au moment où de nouveaux pas effacent les traces des discours antérieurs afin de créer des langages neufs qui incluent dorénavant l'ordre, la pensée et la culture au féminin. 